\title{
The association of diabetes-related self-care activities with perceived stress, anxiety, and fatigue: a cross-sectional study
}

This article was published in the following Dove Press journal:

Patient Preference and Adherence

\author{
Fang-Fang Zhao',2 \\ Riitta Suhonen ${ }^{1,3}$ \\ Jouko Katajisto ${ }^{4}$ \\ Helena Leino-Kilpi ${ }^{1,5}$ \\ 'Department of Nursing Science, \\ Faculty of Medicine, University of \\ Turku, Turku, Finland; ${ }^{2}$ School of \\ Nursing Science, Nantong University, \\ Nantong, People's Republic of China; \\ ${ }^{3}$ Turku University Hospital and City of \\ Turku, Welfare Division, Turku, Finland; \\ ${ }^{4}$ Department of Mathematics and \\ Statistics, University of Turku, Turku, \\ Finland; ${ }^{5}$ Turku University Hospital, \\ Turku, Finland
}

\begin{abstract}
Purpose: Many people with type 2 diabetes (T2DM) do not sustain sufficient diabetes-related self-care activities (DRSCA) in their daily lives. To provide additional information about the positive influence of DRSCA, this study was conducted to examine whether DRSCA were associated with reduced perceived stress, anxiety, and fatigue among people with T2DM and to explore the level of DRSCA, perceived stress, anxiety, and fatigue and their association with background information.
\end{abstract}

Patients and methods: This study was a cross-sectional survey including 251 participants aged 18 years and older recruited from two hospitals in the eastern part of China. The study utilized self-report questionnaires that consisted of background information, DRSCA, perceived stress, anxiety, and fatigue. Hierarchical multiple regression analysis was conducted to explore the association of DRSCA with perceived stress, anxiety, and fatigue while adjusting for background information.

Results: The results indicated that the level of self-care activities, stress, and fatigue was around middle level. The prevalence of anxiety was 19\%. A high level of DRSCA was likely to reduce perceived stress but was not linked to anxiety and fatigue. Women were more susceptible to stress and anxiety, and people who had diabetes for $>5$ years were more likely to have anxiety. The background information included diabetes duration, standardized diabetes education, and high social support, all of which are factors that may influence DRSCA.

Conclusion: The findings suggest that improving the level of DRSCA might effectively reduce perceived stress. The potential benefits of DRSCA can provide both motivational and evaluative data for self-care programs. In addition, the findings show that DRSCA were not linked to anxiety and fatigue, which implies that their positive influence on anxiety and fatigue may be offset by the load of frequent DRSCA. It is suggested that helping patients to make tailored plans to integrate DRSCA into their daily lives is needed. Meanwhile, in the background information, it is suggested that standardized diabetes education and high social support can benefit DRSCA; in improving psychological health, more attention should be paid to women and patients with diabetes duration $>5$ years.

Keywords: diabetes, self-care, adherence, benefits, negative feelings

\section{Introduction}

Diabetes is a major public health problem in the 21 st century. ${ }^{1}$ In China, type 2 diabetes (T2DM) affects $9.9 \%$ of men and $11.6 \%$ of women. ${ }^{2}$ This is slightly higher than the global prevalence of diabetes, which was one in 11 in $2015 .{ }^{3}$ Globally, China accounts for a large percentage of DM cases, which is a critical and costly issue. The majority of diabetes treatment relies on self-care activities. ${ }^{4}$ Many studies have 
indicated that the successful management of diabetes relies on sufficient diabetes-related self-care activities (DRSCA) to control disease progression. ${ }^{5}$ However, it is not an easy task for people with T2DM to perform DRSCA daily and to incorporate DRSCA into their daily lives. ${ }^{6}$ This was seen, for example, in a cross-sectional study conducted in 15 Chinese cities showing that only $13.6 \%$ of the patients achieved glycosylated hemoglobin (HbAlc) $<6.5 \% .^{7}$ In order to promote the overall well-being of patients with T2DM, it is important for healthcare staff to focus on both DRSCA and health. ${ }^{8}$ The most cited DRSCA included, but were not limited to, medication adherence, regulating diet, physical activity, blood glucose monitoring, and foot care. ${ }^{9}$ The importance of DRSCA for patients has been noted by a number of researchers. ${ }^{10}$ Although a vast array of factors associated with self-care activities have been identified, ${ }^{10}$ the explanation for the reason why patients fail to perform self-care activities remains insufficient. ${ }^{11}$ Ways to encourage and empower patients with T2DM to increase their DRSCA levels through the provision of support information have therefore been receiving increasing attention. ${ }^{12-16}$

Embracing this philosophy of empowering patients requires that patients are knowledgeable and active collaborators in their own care. ${ }^{15,16}$ In addition, patients should be supported to make informed decisions to achieve their self-care goals. ${ }^{15}$ Consistent with this approach, additional information about the influence of DRSCA is needed to support patients in diabetes care. A meta-analysis confirmed that perceived benefits, as one important component of the health belief model, ${ }^{17}$ were significantly associated with better DRSCA. ${ }^{18}$ Of note, the evidence shows a paucity of published studies about motivational information encouraging DRSCA. ${ }^{10}$ Besides the benefits in blood glucose control, ${ }^{5}$ DRSCA may have other positive effects and may help to reduce psychological distress and improve physical health by a regular lifestyle. Among people with diabetes, perceived stress, anxiety, and fatigue are common. ${ }^{19-21}$ These individuals have higher perceived stress levels than the general population. ${ }^{21}$ People with diabetes are also likely to have clinical anxiety and experience subclinical anxiety, the prevalence of anxiety being 14\%, and elevated anxiety symptoms $40 \%{ }^{20}$ Furthermore, the prevalence of fatigue is high and has been documented in $61 \%$ of people with diabetes. ${ }^{19}$ As perceived stress, anxiety, and fatigue are common among people with T2DM and they have to perform DRSCA daily, every effort to encourage performing DRSCA is needed. Sufficient DRSCA can lead to improved physical health ${ }^{5}$ and may also influence psychological health. A comprehensive understanding of the association between DRSCA and negative feelings such as perceived stress, anxiety, and fatigue would provide new information for diabetes care. This new and supportive information may help patients be more responsible and self-determined in their daily self-care.

In the present study, DRSCA are assumed to be associated with perceived stress. Perceived stress is defined as the degree to which individuals appraise situations as stressful in their lives when the demands exceed the individuals' ability to cope with them. ${ }^{22}$ In people with diabetes, the disease itself is an additional stressor. Perceived stress can protect the body in the short term but affects the immune system in the long run and has been widely linked to poor health outcomes. ${ }^{23}$ It was recently suggested that self-care-oriented behavior could be a way to improve the ability to manage stress in health promotion. ${ }^{24}$ Despite the recommendation, ${ }^{24}$ there is little evidence available regarding the association between DRSCA and perceived stress. Recent studies have indicated that occupation ${ }^{25}$ and physical activity ${ }^{26}$ are associated with perceived stress. In addition, exploring whether actively taking initiative to conduct DRSCA is associated with reduced stress can provide important information for diabetes stress management.

In the present study, DRSCA are assumed to be associated with anxiety. Anxiety disorder is defined by sleeping problems, behavioral disturbance, excessive worry, irritability, and muscle tension. ${ }^{27}$ Diabetes itself may induce anxiety as individuals perceive the necessity of unwanted lifestyle changes and the risk of diabetes-related complications. ${ }^{28}$ Failure to reach target blood glucose levels is therefore associated with psychological distress. ${ }^{29}$ The intended influence of self-care activities includes a decrease in the anxiety that is often associated with chronic illness. ${ }^{30}$ This implies that performance of DRSCA may relieve psychological distress, such as anxiety. However, there is a lack of evidence as to whether DRSCA are actually associated with reduced anxiety.

In the present study, DRSCA are assumed to be associated with fatigue. Fatigue is defined as a decrease in the capacity to perform physical and/or mental tasks. ${ }^{29}$ Uncontrolled blood glucose levels are indicated to be indirectly associated with fatigue in patients. ${ }^{31}$ Moreover, the severity of diabetes symptoms $^{29}$ and blood glucose level ${ }^{32,33}$ are strongly associated with fatigue. It seems that DRSCA showing benefit for blood glucose level and diabetes symptoms may associate with fatigue. Therefore, it is often assumed that fatigue is diminished by following the diabetes treatment regimen and keeping the disease under control. This has not been empirically tested, however. ${ }^{34}$ 
In view of understanding the role of DRSCA on perceived stress, anxiety, and fatigue, which might benefit future efforts to promote DRSCA, this study aimed to 1) evaluate the level of DRSCA, perceived stress, anxiety, and fatigue among patients with T2DM; 2) evaluate the possible association between participants' background information and DRSCA, perceived stress, anxiety, and fatigue; and 3) evaluate whether DRSCA is associated with stress, anxiety, and fatigue.

\section{Patients and methods Study design and participants}

A descriptive and cross-sectional survey with self-report questionnaires was adopted using convenience sampling for data collection. To participate in the survey, participants had to be 18 years or older and diagnosed with $\mathrm{T} 2 \mathrm{DM} \geq 3$ months and without serious complications or cognition impairment. Participation was voluntary, and the participants provided written informed consent. The sample size calculated for multiple regression analysis (fixed model, $R^{2}$ increase) was 230 , with an alpha of 0.05 , the power of 0.90 , the effect size set at 0.10 , and the number of tested predicted factors and the total number of predicted factors set at 12 in $G^{*}$ Power statistical software. ${ }^{35}$

\section{Ethical consideration}

The study was approved by the ethics committee of a university-affiliated hospital in China in 2015 (120/2015). Permission for data collection was granted by hospital administrators. Participants gave written informed consent before enrollment once the explanation of the objective of the study and the anonymity of data were ensured. The permission to use the instruments was obtained from the copyright holders via email where permission for use was required.

\section{Data collection}

The study was conducted in two tertiary-level universityaffiliated hospitals in Nantong city, eastern China. Data were collected over the period from summer 2015 to summer 2016. Researchers and/or research assistants administered the surveys in Mandarin or in local dialect to adults with T2DM recruited from the hospitals by convenient sampling. Before carrying out the survey, the research assistants were trained to follow standardized procedures. The training for the research assistants included information about the survey, its objectives, the meaning of the questions, the procedure, interviewing techniques, ethical issues, and questionnaire coding. If needed, the questionnaire items were read out and explained to the participants by the investigators.
The participants were given daily necessities such as bars of soap to thank them for their time. Three hundred ten persons agreed to take part in the survey (response rate $\sim 70 \%$ ). After excluding incomplete questionnaires and those reporting only hospital care as past week's self-care activities, 251 valid completed questionnaires were included in the study analysis.

\section{Measures}

\section{Background information}

The background information included age (in years), sex (female/male), education (less than college education/ associate degree or advanced), family history of diabetes (yes/no), experience of symptoms before diagnosis (yes/no), duration of diabetes (in years), and whether the subject had received standard diabetes education before the survey (yes/no) and social support. Social support was measured using the Social Support Scale ${ }^{36}$ including 10 items and the following three components: subjective support, objective support, and utilization of support. One item of the scale has five questions, and the score was summarized from the five questions. Each question was scored from 1 to 4 . Two items were scored from 0 to 9 according to the number of support resources. A higher score indicates a higher level of social support. The scale has good reliability and validity. ${ }^{36}$ Social support scores $\leq 44$ are defined as low level, and scores $>44$ are defined as high level. ${ }^{37}$

\section{Diabetes-related self-care activities}

DRSCA were measured using the Chinese version of the Diabetes Self-Management scale. ${ }^{38}$ Developed based on the diabetes DRSCA scale, ${ }^{9}$ it measures the level of DRSCA. It includes five dimensions: regulating diet, physical activities (continuous physical activity such as housework and/or walking for $>30$ minutes per day), medications, self-monitoring of blood sugar, and foot care. Each item is scored from 0 to 7 , a higher score indicating a higher level of self-care. The eightitem version was used, and five factors explained $>90 \%$ of the variance. ${ }^{38}$

\section{Perceived stress}

Perceived stress was measured by the 10-item Perceived Stress Scale (PSS-10), a short version of the 14-item PSS-14 developed by Cohen et al. ${ }^{22}$ It was designed to measure the degree to which situations in ones' life are appraised as stressful. $^{22}$ The scale options were scored from 0 (never) to 4 (very often). A higher PSS-10 score indicates higher perceived stress. Cronbach's $\alpha$ of the Chinese version of 
PSS-10 was 0.86 , the test-retest reliability was 0.68 , and the exploratory factor analysis yielded two factors accounting for $62.41 \%$ of the variance. ${ }^{39}$

\section{Anxiety}

Anxiety was measured by the Zung Self-Rating Anxiety Scale (SAS) with 20 items forming a single dimension. ${ }^{40}$ For each item, the response options were from " $1=$ none or a little of the time," to " $4=$ most of the time." The raw score (range 20-80) was converted into an "Anxiety Index" score by multiplying by 1.25 . The commonly used cutoff score for diagnosing clinical anxiety is $50 .{ }^{41}$ The scale had split-half reliability $0.71^{40}$ and good concurrent and convergent validity and discriminant validity. ${ }^{42}$ The Chinese version of the Anxiety Scale is a valid instrument for use with Chinese samples. ${ }^{43}$

\section{Fatigue}

The 14-item Fatigue scale including two dimensions of physical fatigue and mental fatigue ${ }^{44}$ was used to measure the severity of fatigue. A Mandarin Chinese version of the 14-item Fatigue scale was used. In our study, a bimodal $(0-1)$ scale was used instead of a 4-point (0-3) Likert scale in rating all items. Specifically, the responses were scored from 0 (none) to 1 (yes), while items 10, 13, and 14 were scored reversely, a higher score reflecting more serious fatigue. The scale was found to be both reliable and valid. Cronbach's $\alpha$ for all items ranged from 0.88 to 0.90 . The scale demonstrated good sensitivity and specificity using an arbitrary cutoff score of $3 / 4 .{ }^{44}$ The Chinese version of the fatigue scale is reliable and valid among Chinese adults in the general population..$^{45,46}$

\section{Statistical analysis}

Data were analyzed using the SPSS 23.0 (IBM Corporation, Armonk, NY, USA). Errors such as data transfer errors and outliers were removed from the data. ${ }^{47}$ The excluded outliers lie outside the range Q1 (first quartile) $-1.5 \times$ interquartile range (IQR) or Q3 (third quartile) $+1.5 \times \mathrm{IQR}$. The number of missing values in study variables in the valid questionnaires was $<2 \%$ and was imputed using the expectation-maximization method. In the data analysis, descriptive statistics including percentage, mean, and SD was presented to describe the data. Cronbach's $\alpha$ s were calculated for the scales. Independent sample $t$-test was conducted to explore the association of background information with perceived stress, anxiety, and fatigue, and Pearson's correlation analysis was used to reflect correlation between the main study variables.
Hierarchical multiple regression analysis was used to explore the associations between DRSCA and perceived stress, anxiety, and fatigue while adjusting for background information. In step 1, the covariates included in the significant background information based on $t$-test on perceived stress, anxiety, and fatigue were adjusted for. In step 2, the significant association between perceived stress, anxiety, and fatigue in correlation analysis was entered. In step 3, the DRSCA were added to the model. Standardized coefficients were computed to present the strength of association between the variables in the regression model. A $P<0.05$ was considered to indicate statistical significance. $R^{2}$ values for the dependent variables represented the amount of variance explained.

\section{Results \\ Background information}

After excluding three outliers, the analysis was run on a data set with 248 questionnaires. The age of the participants ranged from 22 to 85 years $(M=59.5, \mathrm{SD}=13.3)$, with $38.3 \% \geq 65$ years. About half were men (56\%). The average diabetes duration was $\sim 10$ years $(M=9.9, \mathrm{SD}=8.3$, range $0.5-37$ ), and $68.1 \%$ of the participants had diabetes $\geq 5$ years. Less than half $(43.5 \%)$ had not experienced symptoms before diagnosis, and the majority of the participants did not have a family history of diabetes $(73.4 \%)$. Only $25 \%$ had college education or above, and $42.7 \%$ of the participants had received standard diabetes education before the survey. About half (48\%) of the participants felt that they had high social support (Table 1).

\section{The description of DRSCA, perceived stress, anxiety, and fatigue}

In the study population, the score of DRSCA was slightly higher than middle level, the perceived stress level was slightly below middle level, and fatigue was at middle level. The standard score for anxiety was $<50$. Based on the diagnosis cutoff value in China, the score showed that most participants did not have clinical anxiety (19\% possibly having clinical anxiety in this study). However, the anxiety score may still be used as a tool to evaluate the degree of anxiety. The Cronbach's $\alpha$ s for the scales, ranging from 0.64 to 0.9 , were also reported to be acceptable (Table 2). ${ }^{48}$

\section{Association between background information and perceived stress, anxiety, and fatigue}

In our study, women and participants who had diabetes for $>5$ years were more likely to have anxiety and women 
Table I Background information of the participants

\begin{tabular}{ll}
\hline Characteristics & $\mathbf{n}(\%)$ \\
\hline Sex & \\
$\quad$ Male & $139(56)$ \\
Female & $109(44)$ \\
Age (years) & \\
$\quad<65$ & $153(61.7)$ \\
$\geq 65$ & $95(38.3)$ \\
Diabetes duration (years) & \\
$\quad<5$ & $79(31.9)$ \\
$\geq 5$ & $169(68.1)$ \\
Had symptoms before diagnosis & \\
$\quad$ Yes & $140(56.5)$ \\
No & $108(43.5)$ \\
Education & \\
$\quad$ Less than college education & $186(75.0)$ \\
Associate degree or advanced & $62(25.0)$ \\
Received SSCE & \\
None & $142(57.3)$ \\
Yes & $106(42.7)$ \\
Living alone & \\
Yes & $56(22.6)$ \\
No & $192(77.4)$ \\
Family history of diabetes & \\
Yes & $66(26.6)$ \\
No & $182(73.4)$ \\
Social support & \\
Low & $129(52)$ \\
High & $119(48)$ \\
\hline
\end{tabular}

Abbreviation: SSCE, standardized self-care education.

were also likely to have perceived stress. Participants who had diabetes for $>5$ years, had received standardized diabetes education, and had high social support were more likely to have better DRSCA (Table 3).

\section{Correlation between DRSCA, perceived stress, anxiety, and fatigue}

Pearson correlation coefficients showed that DRSCA had a significant negative correlation with perceived stress. The correlation between the perceived stress and anxiety was positive, as was the correlation between anxiety and fatigue (Table 4).

Table 2 The description of DRSCA, perceived stress, anxiety and fatigue $(\mathrm{n}=248)$

\begin{tabular}{llllll}
\hline Variables & Mean & SD & $\begin{array}{l}\text { Possible } \\
\text { range }\end{array}$ & $\begin{array}{l}\text { Score } \\
\text { index (\%) }\end{array}$ & $\begin{array}{l}\text { Cronbach's } \alpha \\
\text { in this study }\end{array}$ \\
\hline DRSCA & 34.9 & 11.2 & $0-56$ & 62 & 0.64 \\
Perceived stress & 14.1 & 6.7 & $0-40$ & 35 & 0.90 \\
Anxiety & 40.8 & 8.5 & $25-100$ & $4 I$ & 0.78 \\
Fatigue & 7.2 & 3.9 & $0-14$ & 51 & 0.87 \\
\hline
\end{tabular}

Notes: Score index, actual score divided by the possible maximum score. Possible range is the range of the scale total score.

Abbreviation: DRSCA, diabetes-related self-care activities.

\section{Hierarchical multiple regression analysis for the associations between study variables}

Hierarchical multiple regression analysis was used to analyze the association between DRSCA and perceived stress, anxiety, and fatigue. Covariates for the analysis were chosen based on the statistical significance level from $t$-test and Pearson's correlation analysis (Table 5).

For the dependent variable perceived stress, at step 1, sex was significantly associated with perceived stress $(\beta=0.149$, $P=0.019)$. At step 2 , anxiety was introduced and was significantly associated with stress $(\beta=0.374, P<0.001)$. At step 3 , DRSCA were significantly associated with perceived stress after adjusting for sex and anxiety $(\beta=-0.220, P<0.001)$. The model explained $20.2 \%$ of the variance in perceived stress. Excluding the influence of demographics and anxiety, DRSCA explained an additional significant $4.8 \%$ of the variance.

For the dependent variable anxiety, at step $1, \operatorname{sex}(\beta=0.239$, $P<0.001)$ and diabetes duration $(\beta=0.125, P=0.043)$ were significantly associated with anxiety. At step 2 , perceived stress and fatigue were entered; perceived stress $(\beta=0.375$, $P<0.001)$ and fatigue $(\beta=0.213, P<0.001)$ were significantly associated with anxiety. At step 3, DRSCA were not significantly associated with anxiety after adjusting for sex, diabetes duration, perceived stress, and fatigue ( $\beta=0.049, P=0.402$ ). The variance of anxiety explained by the model was $25.4 \%$. Excluding the influence of demographics and fatigue, DRSCA were not significantly associated with anxiety.

For the dependent variable fatigue, at step 1 , anxiety was significantly associated with fatigue $(\beta=0.207, P=0.001)$. At step 2, DRSCA were not associated with fatigue ( $\beta=-0.031, P=0.623$ ). The model explained $4.4 \%$ of the variance in fatigue. Excluding the influence of anxiety, DRSCA were not significantly associated with fatigue.

\section{Discussion}

The results of the study showed that the score of DRSCA, perceived stress, and fatigue was around middle level, and the anxiety value indicated that most participants did not have clinical anxiety. The perceived stress score index in this population was similar to a previous study conducted in India, ${ }^{49}$ the fatigue level corresponded with a previous report in people with $\mathrm{T} 2 \mathrm{DM},{ }^{50}$ and the prevalence of anxiety corresponded with a previous report ${ }^{51}$ but was lower than that reported in other studies. ${ }^{43,52}$ The score index of DRSCA in the study was similar to the results of some other studies. ${ }^{53,54}$ Although the score index of DRSCA was around middle level, it was still higher than in other studies, ${ }^{55-57}$ indicating 
Table 3 The association of background information with perceived stress, anxiety, and fatigue $(n=248)$

\begin{tabular}{|c|c|c|c|c|c|c|c|c|c|}
\hline & \multirow[t]{2}{*}{ n (\%) } & \multicolumn{2}{|l|}{ DRSCA } & \multicolumn{2}{|c|}{ Perceived stress } & \multicolumn{2}{|l|}{ Anxiety } & \multicolumn{2}{|l|}{ Fatigue } \\
\hline & & Mean \pm SD & $P(t)$ & Mean \pm SD & $P(t)$ & Mean \pm SD & $P(t)$ & Mean \pm SD & $P(t)$ \\
\hline Sex & & & 0.354 & & 0.019 & & 0.000 & & 0.889 \\
\hline Male & $139(56)$ & $34.4 \pm I I . I$ & & $13.2 \pm 6.7$ & & $39.0 \pm 8.7$ & & $7.2 \pm 3.9$ & \\
\hline Female & $109(44)$ & $35.7 \pm 11.4$ & & $15.2 \pm 6.6$ & & $43.2 \pm 7.7$ & & $7.2 \pm 3.9$ & \\
\hline Age (years) & & & 0.086 & & 0.068 & & 0.694 & & 0.549 \\
\hline$<65$ & $153(61.7)$ & $34.0 \pm 11.7$ & & $14.7 \pm 6.8$ & & $40.7 \pm 8.4$ & & $7.1 \pm 3.8$ & \\
\hline$\geq 65$ & $95(38.3)$ & $36.5 \pm 10.4$ & & $|3| \pm 6.4$. & & $4 I . I \pm 8.7$ & & $7.4 \pm 4.0$ & \\
\hline Diabetes duration (years) & & & 0.002 & & 0.559 & & 0.032 & & 0.354 \\
\hline$<5$ & $79(31.9)$ & $31.7 \pm 12.0$ & & $14.4 \pm 6.7$ & & $39.1 \pm 8.1$ & & $6.8 \pm 4.0$ & \\
\hline$\geq 5$ & $169(68.1)$ & $36.5 \pm 10.6$ & & $13.9 \pm 6.7$ & & $41.6 \pm 8.6$ & & $7.3 \pm 3.8$ & \\
\hline Had symptoms before diagnosis & & & 0.187 & & 0.975 & & 0.596 & & 0.658 \\
\hline Yes & $140(56.5)$ & $34.2 \pm 11.8$ & & $\mid 4.1 \pm 6.7$ & & $40.6 \pm 8.1$ & & $7.1 \pm 3.5$ & \\
\hline No & $108(43.5)$ & $36.1 \pm 10.5$ & & $14.0 \pm 6.7$ & & $4 I . I \pm 8.9$ & & $7.3 \pm 4.3$ & \\
\hline Education & & & 0.145 & & 0.182 & & 0.819 & & 0.147 \\
\hline Less than college education & $186(75.0)$ & $34.4 \pm \mathrm{II} . \mathrm{I}$ & & $14.4 \pm 6.6$ & & $40.9 \pm 8.3$ & & $7.4 \pm 3.8$ & \\
\hline Associate degree or advanced & $62(25.0)$ & $36.8 \pm I I .8$ & & $13.1 \pm 6.9$ & & $40.6 \pm 9.0$ & & $6.6 \pm 3.9$ & \\
\hline Received SSCE & & & 0.021 & & 0.946 & & 0.642 & & 0.109 \\
\hline None & $142(57.3)$ & $33.6 \pm I I .4$ & & $14.0 \pm 7.5$ & & $41.0 \pm 9.2$ & & $7.5 \pm 3.9$ & \\
\hline Yes & $106(42.7)$ & $36.9 \pm 10.8$ & & $|4| \pm 5.6$. & & $40.5 \pm 7.4$ & & $6.7 \pm 3.8$ & \\
\hline Living alone & & & 0.069 & & 0.821 & & 0.635 & & 0.850 \\
\hline Yes & $56(22.6)$ & $32.6 \pm I I .4$ & & $14.2 \pm 6.0$ & & $41.3 \pm 8.2$ & & $7.3 \pm 3.7$ & \\
\hline No & $192(77.4)$ & $35.7 \pm 11.1$ & & $14.0 \pm 6.9$ & & $40.7 \pm 8.6$ & & $7.1 \pm 3.9$ & \\
\hline Family history of diabetes & & & 0.169 & & 0.925 & & 0.878 & & 0.118 \\
\hline Yes & $66(26.6)$ & $33.3 \pm 11.5$ & & $14.0 \pm 6.9$ & & $41.0 \pm 8.5$ & & $7.8 \pm 3.8$ & \\
\hline No & I $82(73.4)$ & $35.6 \pm 11.1$ & & $\mid 4.1 \pm 6.7$ & & $40.8 \pm 8.5$ & & $7.0 \pm 3.9$ & \\
\hline Social support & & & 0.007 & & 0.966 & & 0.545 & & 0.906 \\
\hline Low & $129(52)$ & $33.1 \pm I I .5$ & & $14.1 \pm 6.5$ & & $4 I . I \pm 8.9$ & & $7.2 \pm 4.0$ & \\
\hline High & $119(48)$ & $37.0 \pm 10.7$ & & $14.0 \pm 7.0$ & & $40.5 \pm 8.0$ & & $7.1 \pm 3.8$ & \\
\hline
\end{tabular}

Note: The $P$-values are bold when they are $<0.05$.

Abbreviations: DRSCA, diabetes related self-care activities; SSCE, standardized self-care education.

that the level of DRSCA was in general not optimistic. However, no clear conclusions can be drawn in comparison with previous studies; the discrepancies may be due to differences in the patients' cultural background and situation.

Table 4 Pearson's correlation coefficients between the study variables $(n=248)$

\begin{tabular}{|c|c|c|c|c|}
\hline & DRSCA & $\begin{array}{l}\text { Perceived } \\
\text { stress }\end{array}$ & Anxiety & Fatigue \\
\hline \multicolumn{5}{|l|}{ DRSCA } \\
\hline Pearson's $r$ & I & & & \\
\hline \multicolumn{5}{|l|}{$P$-value } \\
\hline \multicolumn{5}{|c|}{ Perceived stress } \\
\hline Pearson's $r$ & $-0.219 *$ & I & & \\
\hline$P$-value & 0.001 & & & \\
\hline \multicolumn{5}{|l|}{ Anxiety } \\
\hline Pearson's $r$ & -0.008 & $0.388^{*}$ & I & \\
\hline$P$-value & 0.900 & 0.000 & & \\
\hline \multicolumn{5}{|l|}{ Fatigue } \\
\hline Pearson's $r$ & -0.032 & -0.041 & $0.207^{*}$ & I \\
\hline$P$-value & 0.611 & 0.518 & 0.001 & \\
\hline
\end{tabular}

Note: *Correlation is significant at the 0.01 level (two tailed).

Abbreviation: DRSCA, diabetes-related self-care activities.
In the first step of the hierarchical regression analyses, we found that sex was associated with perceived stress and anxiety while diabetes duration was associated with anxiety. Women were more likely to experience stress and to be at risk of anxiety, in line with previous findings. ${ }^{43}$ This finding may possibly be explained by the gender-specific roles and social role of women, which may expose them to more stress and more emotional burden compared with men. ${ }^{52}$ The independent samples' $t$-test results showed that participants who had diabetes for $>5$ years, had received standardized diabetes education, and had high social support were more likely to have better DRSCA. The role of social support on DRSCA is supported by previous studies. ${ }^{58,59}$ Long-term disease ( $>5$ years duration) was significantly associated with higher anxiety scores. The result was consistent with previous studies. ${ }^{43,60}$ The possible reason may be that chronic disease significantly increases the risk of developing diabetic complications and increases healthcare expenditure; ${ }^{61}$ as a result, such patients are more likely to develop psychological illnesses ${ }^{43}$ such as anxiety. However, this study showed that patients with longer diabetes duration had more experiences 
Table 5 Hierarchical multiple regression analysis of the association between DRSCA and perceived stress, anxiety, and fatigue $(n=248)$

\begin{tabular}{|c|c|c|c|c|c|c|c|}
\hline \multirow[t]{2}{*}{ DV } & \multirow[t]{2}{*}{ IV } & \multicolumn{2}{|l|}{ Step I } & \multicolumn{2}{|l|}{ Step 2} & \multicolumn{2}{|l|}{ Step 3} \\
\hline & & $\beta$ (SE) & $P$-value & $\beta$ (SE) & $P$-value & $\beta$ (SE) & $P$-value \\
\hline \multirow[t]{3}{*}{ Perceived stress } & Sex & $0.149(0.852)$ & 0.019 & $0.058(0.819)$ & $0.34 I$ & $0.072(0.799)$ & 0.224 \\
\hline & Anxiety & & & $0.374(0.048)$ & $<0.001$ & $0.369(0.047)$ & $<0.001$ \\
\hline & DRSCA & & & & & $-0.220(0.034)$ & $<0.001$ \\
\hline \multirow[t]{5}{*}{ Anxiety } & Sex & $0.239(1.050)$ & $<0.001$ & 0.181 (0.959) & 0.001 & $0.177(0.964)$ & 0.002 \\
\hline & Diabetes duration & $0.125(1.118)$ & 0.043 & $0.129(1.013)$ & 0.021 & $0.120(1.033)$ & 0.036 \\
\hline & Perceived stress & & & $0.375(0.07 I)$ & $<0.001$ & $0.386(0.073)$ & $<0.001$ \\
\hline & Fatigue & & & $0.213(0.122)$ & $<0.001$ & $0.216(0.123)$ & $<0.001$ \\
\hline & DRSCA & & & & & $0.049(0.044)$ & 0.402 \\
\hline \multirow[t]{2}{*}{ Fatigue } & Anxiety & $0.207(0.028)$ & 0.001 & $0.207(0.028)$ & 0.001 & & \\
\hline & DRSCA & & & $-0.03 \mathrm{I}(0.02 \mathrm{I})$ & 0.623 & & \\
\hline
\end{tabular}

Note: $\beta$, standardized coefficients.

Abbreviations: DRSCA, diabetes-related self-care activities; DV, dependent variables; IV, independent variables; SE, standard errors.

and showed better levels of DRSCA, which may indirectly relieve anxiety through the link from improved DRSCA to reduced stress.

Unlike previous studies, ${ }^{51,62,63}$ we also found that social support had no association with perceived stress, anxiety, and fatigue. The connection with social support may be mediated through other factors. A previous study showed that social support was associated with subsequent mental health through resilience. ${ }^{64}$ Another explanation was that social support may tend to be associated with higher levels of stress, anxiety, and fatigue, which were not high in this population. However, further exploration of their levels of relevance may be warranted.

The study indicates that a high level of DRSCA was significantly associated with lowered perceived stress, confirming the earlier suggestions that self-care could reduce perceived stress. ${ }^{24}$ Stress can cause blood glucose to increase or to become unstable, which is why reducing perceived stress would be beneficial for patients with diabetes. Empowering patients to persist with DRSCA is important in diabetes management. ${ }^{56}$ Setting measurable and achievable goals for daily DRSCA may be helpful in terms of motivating people and minimizing perceived stress. This finding implies that stress can be an outcome evaluated in diabetes management. In addition, performing DRSCA is a positive coping method to reduce perceived stress among patients. Thus, it is suggested that the consequences of performing DRSCA may well extend beyond blood glucose control and have positive influence on reducing perceived stress. However, this finding needs to be confirmed in further interventional studies. The findings also demonstrated that perceived stress correlates with anxiety, which is consistent with the study of Zhang et al..$^{51}$ The average age of participants and the percentage of college education level in this study were similar to a previous study. ${ }^{51}$ People with more education seemed more likely to be employed and find a job providing more healthpromoting benefits such as health insurance, paid leave, and retirement. ${ }^{65}$ A previous study showed socioeconomic status to be linked to mood disorders and anxiety. ${ }^{66}$ Therefore, education level may indirectly influence stress and anxiety through financial support for health and wellness while health insurance may be directly associated with stress and anxiety. In this study, we did not investigate the participants' socioeconomic status and health insurance in detail, which may have an influence on stress and anxiety. In future, it is suggested that new research should be undertaken to shed light on these influences.

In this study, we also found that DRSCA did not associate with reduced anxiety. A reason for this may be that depression ${ }^{25,55}$ and perceived stress ${ }^{51}$ account for a significant contribution to anxiety. Another potential explanation for the insignificant statistical association may be that DRSCA imposed some restrictions on patients' lives, which may have offset the positive influence of the DRSCA. The present study indicates that DRSCA were associated with reduced perceived stress while perceived stress was associated with anxiety. Therefore, DRSCA may reduce anxiety indirectly. In our study, DRSCA were not directly associated with fatigue. Given that blood glucose control was associated with fatigue, ${ }^{31}$ further research is needed to elucidate whether DRSCA have an indirect influence on fatigue through controlling blood glucose. Fritschi and Quinn ${ }^{29}$ summarized that limited research directly associates emotional distress in diabetes with fatigue. In the present study, anxiety as a kind of emotional distress was shown to be associated with fatigue, which is in accordance with a study among patients with multiple sclerosis. ${ }^{67}$ Besides anxiety, a previous study showed that poor sleep quality ${ }^{31}$ and pain $^{50}$ were 
contributing factors. This finding also suggests the necessity of further exploration of other multifactors associated with anxiety and fatigue among patients with diabetes.

\section{Limitations}

The study had some limitations, and interpretation of results should thus be done within the context of our methods. First, the cross-sectional design of the study does not allow drawing definite conclusions about the causal association between self-care activities and perceived stress, anxiety, and fatigue. However, the results of this study provide some evidence for a future intervention study to confirm the causality of the concepts.

Second, the generalization of the results should be approached with caution. We only surveyed participants in two hospitals in China by convenient sampling; using a wider national sample was not possible due to time and budget limitations. Our none-probability sampling method may impact the representability of the area where the study was conducted. We evaluate the possibility of bias as not substantial as there is a wide age range and close to gender balance in our study: according to medical records, the population with T2DM of these two hospitals generally had a balanced gender ratio and were aged from 19 to $90+$ years. The gender ratio and mean age in this study were also similar to a previous study among patients with T2DM conducted in this area ${ }^{68}$ However, the none-probability sampling is more likely to recruit more cooperative patients without serious disease. Therefore, research with random sampling from multiple districts in China would enable wider generalization of the results in the future.

Third, we did not include HbAlc in our study. Its important association with emotional distress and fatigue has been noted in previous studies. ${ }^{29,32,33}$ The mediating effects of $\mathrm{HbA} 1 \mathrm{c}$ between DRSCA and emotional distress with fatigue call for investigation. We focused only on the association between DRSCA and perceived stress, anxiety, and fatigue. Analysis including HbA1c would be important as a part of large-scale future research.

Fourth, self-report measures may be subjective to response bias and the overestimation of behavior performance. ${ }^{69}$ The sensitivity of the instruments also needs further consideration. Nevertheless, the instruments have been shown to be valid and reliable, and as one of the most feasible data collecting methods, self-report measures can offer actionable information despite the limitations. ${ }^{70}$ Finally, the percentage of variance in the regression model was not high, implying that other associated factors such as socioeconomic status need to be explored further. In spite of the limitations, the study reveals additional information about the association between DRSCA and perceived stress, anxiety, and fatigue, which enriches the understanding of the role of DRSCA among patients with T2DM.

\section{Conclusion}

The findings suggest that improving the level of DRSCA might effectively reduce perceived stress in people with T2DM. As the DRSCA in T2DM are demanding on a daily basis, this information can be used to encourage people to take care of themselves actively. These results can be used in developing empowering educational interventions for this group of people. Although our study showed that DRSCA have no influence on anxiety and fatigue, it does provide potential information for clinical practice to consider. As the cross-sectional design prevents drawing direct causal connections, further interventional studies are needed.

\section{Acknowledgments}

The authors gratefully acknowledge associate professors Li-Hua Zhao and Ai-Juan Wu from affiliated hospitals of Nantong University for their coordinating work in data collection in China. We also thank the research assistants, doctors, and nurses for their help in data collection and the people with diabetes who participated in the study. The study received financial support from CIMO (The Centre for International Mobility) fellowships in Finland. We also thank Anna Vuolteenaho for language revision.

\section{Author contributions}

All authors contributed toward data analysis, drafting and revising the paper and agree to be accountable for all aspects of the work.

\section{Disclosure}

The authors report no conflicts of interest in this work.

\section{References}

1. Unnikrishnan R, Pradeepa R, Joshi SR, Mohan V. Type 2 diabetes: demystifying the global epidemic. Diabetes. 2017;66(6):1432-1442.

2. Yang L, Shao J, Bian Y, et al. Prevalence of type 2 diabetes mellitus among inland residents in China (2000-2014): a meta-analysis. J Diabetes Investig. 2016;7(6):845-852.

3. International Diabetes Federation [webpage on the Internet]. IDF Diabetes Atlas. 7th ed. 2015. Available from: https://www.idf.org/ e-library/epidemiology-research/diabetes-atlas/13-diabetes-atlasseventh-edition.html. Accessed Octorber 20, 2017.

4. Anderson RM, Funnell MM, Butler PM, Arnold MS, Fitzgerald JT, Feste CC. Patient empowerment. Results of a randomized controlled trial. Diabetes Care. 1995;18(7):943-949.

5. Zhou Y, Liao L, Sun M, He G. Self-care practices of Chinese individuals with diabetes. Exp Ther Med. 2013;5(4):1137-1142.

6. Chawla K, Kalra S, Kalra B. Role of psychology in diabetes management. Internet J Endocrinol. 2009;6(1):1-3. 
7. Fu C, Ji L, Wang W, et al. Frequency of glycated hemoglobin monitoring was inversely associated with glycemic control of patients with type 2 diabetes mellitus. J Endocrinol Invest. 2012;35(3):269-273.

8. Sato M, Yamazaki Y. Work-related factors associated with self-care and psychological health among people with type 2 diabetes in Japan. Nurs Health Sci. 2012;14(4):520-527.

9. Toobert DJ, Hampson SE, Glasgow RE. The summary of diabetes self-care activities measure: results from 7 studies and a revised scale. Diabetes Care. 2000;23(7):943-950.

10. Schulman-Green D, Jaser SS, Park C, Whittemore R. A metasynthesis of factors affecting self-management of chronic illness. J Adv Nurs. 2016; 72(7):1469-1489.

11. Blackburn DF, Swidrovich J, Lemstra M. Non-adherence in type 2 diabetes: practical considerations for interpreting the literature. Patient Prefer Adherence. 2013;7:183-189.

12. Linmans JJ, Knottnerus JA, Spigt M. How motivated are patients with type 2 diabetes to change their lifestyle? A survey among patients and healthcare professionals. Prim Care Diabetes. 2015;9(6):439-445.

13. Choi S, Song M, Chang SJ, Kim SA. Strategies for enhancing information, motivation, and skills for self-management behavior changes: a qualitative study of diabetes care for older adults in Korea. Patient Prefer Adherence. 2014;8:219-226.

14. Al-Hassan MA, Al-Akour NA, Aburas MM. Relationship between motivational style and glycemic control in Jordanian patients with type 2 diabetes mellitus. J Diabetes. 2017;9(1):93-101.

15. Funnell MM, Anderson RM. Empowerment and self-management of diabetes. Clin Diabetes. 2004;22(3):123-127.

16. Eyüboğlu E, Schulz PJ. Do health literacy and patient empowerment affect self-care behaviour? A survey study among Turkish patients with diabetes. BMJ Open. 2016;6(3):e010186.

17. Rosenstock IM. Historical origins of the health belief model. Health Educ Monogr. 1974;2(4):328-335.

18. Luo X, Liu T, Yuan X, et al. Factors influencing self-management in Chinese adults with type 2 diabetes: a systematic review and metaanalysis. Int J Environ Res Public Health. 2015;12(9):11304-11327.

19. Drivsholm T, de Fine Olivarius N, Nielsen AB, Siersma V, Symptoms SV. Symptoms, signs and complications in newly diagnosed type 2 diabetic patients, and their relationship to glycaemia, blood pressure and weight. Diabetologia. 2005;48(2):210-214.

20. Grigsby AB, Anderson RJ, Freedland KE, Clouse RE, Lustman PJ Prevalence of anxiety in adults with diabetes: a systematic review. J Psychosom Res. 2002;53(6):1053-1060.

21. Eom YS, Park HS, Kim SH, et al. Evaluation of stress in Korean patients with diabetes mellitus using the problem areas in diabetes-Korea questionnaire. Diabetes Metab J. 2011;35(2):182-187.

22. Cohen S, Kamarck T, Mermelstein R. A global measure of perceived stress. J Health Soc Behav. 1983;24(4):385-396.

23. Pouwer F, Kupper N, Adriaanse MC. Does emotional stress cause type 2 diabetes mellitus? A review from the European Depression in Diabetes (EDID) Research Consortium. Discov Med. 2010;9(45):112-118.

24. Esch T, Fricchione GL, Joos S, Teut M. Self-care, stress management, and primary care: from salutogenesis and health promotion to mind-body medicine. Evid Based Complement Alternat Med. 2013;2013:327415.

25. Tan KC, Chan GC, Eric H, et al. Depression, anxiety and stress among patients with diabetes in primary care: a cross-sectional study. Malays Fam Physician. 2015;10(2):9-21.

26. Sendhilkumar M, Tripathy JP, Harries AD, et al. Factors associated with high stress levels in adults with diabetes mellitus attending a tertiary diabetes care center, Chennai, Tamil Nadu, India. Indian J Endocrinol Metab. 2017;21(1):56-63.

27. Allgulander C. Generalized anxiety disorder: what are we missing? Eur Neuropsychopharmacol. 2006;16(Suppl 2):S101-S108.

28. Bickett A, Tapp H. Anxiety and diabetes: innovative approaches to management in primary care. Exp Biol Med. 2016;241(15):1724-1731.

29. Fritschi C, Quinn L. Fatigue in patients with diabetes: a review. J Psychosom Res. 2010;69(1):33-41.

30. Riegel B, Jaarsma T, Strömberg A. A middle-range theory of self-care of chronic illness. ANS Adv Nurs Sci. 2012;35(3):194-204.
31. Park H, Park C, Quinn L, Fritschi C. Glucose control and fatigue in type 2 diabetes: the mediating roles of diabetes symptoms and distress. J Adv Nurs. 2015;71(7):1650-1660.

32. Jain A, Sharma R, Choudhary PK, Yadav N, Jain G, Maanju M. Study of fatigue, depression, and associated factors in type 2 diabetes mellitus in industrial workers. Ind Psychiatry J. 2015;24(2):179-184.

33. Seo YM, Hahm JR, Hahm JR, Kim TK, Choi WH. Factors affecting fatigue in patients with type II diabetes mellitus in Korea. Asian Nurs Res (Korean Soc Nurs Sci). 2015;9(1):60-64.

34. Goedendorp MM, Tack CJ, Steggink E, Bloot L, Bazelmans E, Knoop H. Chronic fatigue in type 1 diabetes: highly prevalent but not explained by hyperglycemia or glucose variability. Diabetes Care. 2014; 37(1):73-80.

35. Faul F, Erdfelder E, Buchner A, Lang AG [webpage on the Internet]. G*Power Version 3.1.7 [Computer Software]. Germany: Uiversität Kiel; 2013. Available from: http://www.psycho.uni-duesseldorf.de/abteilungen/ aap/gpower3/download-and-register. Accessed July 21, 2018.

36. Xiao SY. The theoretical foundation of the social support rating scale and applications in research. J Clin Psychol Med. 1994;4(2):98-100.

37. Dai W, Chen L, Tan H, et al. Association between social support and recovery from post-traumatic stress disorder after flood: a 13-14 year follow-up study in Hunan, China. BMC Public Health. 2016;16:194.

38. Xu Y, Savage C, Toobert D, Wei P, Whitmer K. Adaptation and testing of instruments to measure diabetes self-management in people with type 2 diabetes in mainland China. J Transcult Nurs. 2008;19(3):234-242.

39. Wang Z. Psychometric properties of the Chinese version of the perceived stress scale in policewomen (Psychometrics of the Chinese PSS). PLoS One. 2011;6(12):e28610.

40. Zung WW. A rating instrument for anxiety disorders. Psychosomatics. 1971;12(6):371-379.

41. Zung WW, Magruder-Habib K, Velez R, Alling W. The comorbidity of anxiety and depression in general medical patients: a longitudinal study. J Clin Psychiatry. 1990;51(Suppl 77-80):discussion 81.

42. Samakouri M, Bouhos G, Kadoglou M, Giantzelidou A, Tsolaki K, Livaditis M. Standardization of the Greek version of Zung's Self-rating Anxiety Scale (SAS). Psychiatriki. 2012;23(3):212-220.

43. Sun N, Lou P, Shang Y, et al. Prevalence and determinants of depressive and anxiety symptoms in adults with type 2 diabetes in China: a cross-sectional study. BMJ Open. 2016;6(8):e012540.

44. Chalder T, Berelowitz G, Pawlikowska T, et al. Development of a fatigue scale. J Psychosom Res. 1993;37(2):147-153.

45. Wong WS, Fielding R. Construct validity of the Chinese version of the Chalder Fatigue Scale in a Chinese community sample. J Psychosom Res. 2010;68(1):89-93.

46. Jing MJ, Wang JJ, Lin WQ, Lei YX, Wang PX. A community-based cross-sectional study of fatigue in middle-aged and elderly women. J Psychosom Res. 2015;79(4):288-294.

47. van den Broeck J, Cunningham SA, Eeckels R, Herbst K. Detecting data cleaning: diagnosing, and editing data abnormalities. PLoS Med. 2005; 2(10):e267.

48. AGREE Collaboration. Development and validation of an international appraisal instrument for assessing the quality of clinical practice guidelines: the AGREE project. Qual Saf Health Care. 2003;12(1):18-23.

49. Siddiqui A, Madhu SV, Sharma SB, Desai NG. Endocrine stress responses and risk of type 2 diabetes mellitus. Stress. 2015;18(5):498-506.

50. Singh R, Teel C, Sabus C, Mcginnis P, Kluding P. Fatigue in type 2 diabetes: impact on quality of life and predictors. PLoS One. 2016; 11(11):e0165652.

51. Zhang CX, Chen YM, Chen WQ. Association of psychosocial factors with anxiety and depressive symptoms in Chinese patients with type 2 diabetes. Diabetes Res Clin Pract. 2008;79(3):523-530.

52. Khuwaja AK, Lalani S, Dhanani R, Azam IS, Rafique G, White F. Anxiety and depression among outpatients with type 2 diabetes: a multicentre study of prevalence and associated factors. Diabetol Metab Syndr. 2010;2:72.

53. Yang L, Wu Q, Hao Y, et al. Self-management behavior among patients with diabetic retinopathy in the community: a structural equation model. Qual Life Res. 2017;26(2):359-366. 
54. Simon-Tuval T, Shmueli A, Harman-Boehm I. Adherence to self-care behaviors among patients with type 2 diabetes-the role of risk preferences. Value Health. 2016;19(6):844-851.

55. Wu SF, Huang YC, Liang SY, Wang TJ, Lee MC, Tung HH. Relationships among depression, anxiety, self-care behaviour and diabetes education difficulties in patients with type- 2 diabetes: a cross-sectional questionnaire survey. Int J Nurs Stud. 2011;48(11):1376-1383.

56. Yang S, Hsue C, Lou Q. Does patient empowerment predict self-care behavior and glycosylated hemoglobin in Chinese patients with type 2 diabetes? Diabetes Technol Ther. 2015;17(5):343-348.

57. Gao J, Wang J, Zheng P, et al. Effects of self-care, self-efficacy, social support on glycemic control in adults with type 2 diabetes. BMC Fam Pract. 2013;14:66.

58. Miller TA, Dimatteo MR. Importance of family/social support and impact on adherence to diabetic therapy. Diabetes Metab Syndr Obes. 2013;6:421-426.

59. Schiøtz ML, Bøgelund M, Almdal T, Jensen BB, Willaing I. Social support and self-management behaviour among patients with type 2 diabetes. Diabet Med. 2012;29(5):654-661.

60. Selvarajah D, Cash T, Sankar A, et al. The contributors of emotional distress in painful diabetic neuropathy. Diab Vasc Dis Res. 2014;11(4): $218-225$.

61. Khowaja LA, Khuwaja AK, Cosgrove P. Cost of diabetes care in outpatient clinics of Karachi, Pakistan. BMC Health Serv Res. 2007;7:189.

62. Makabe R, Nomizu T. Social support and psychological and physical states among Japanese women with breast cancer before and after breast surgery. Oncol Nurs Forum. 2007;34(4):883-889.
63. Aylaz R, Karadağ E, Işik K, Yildirim M. Relationship between social support and fatigue in patients with type 2 diabetes mellitus in the east of Turkey. Jpn J Nurs Sci. 2015;12(4):367-376.

64. Koelmel E, Hughes AJ, Alschuler KN, Ehde DM. Resilience mediates the longitudinal relationships between social support and mental health outcomes in multiple sclerosis. Arch Phys Med Rehabil. 2017; 98(6):1139-1148

65. Baum S, Ma J, Payea K. The Benefits of Higher Education for Individuals and Society. Trends in Higher Education Series. New York: College Board; 2013:2013.

66. Mucci N, Giorgi G, Roncaioli M, Fiz Perez J, Arcangeli G. The correlation between stress and economic crisis: a systematic review. Neuropsychiatr Dis Treat. 2016;12:983-993.

67. Labuz-Roszak B, Kubicka-Bączyk K, Pierzchała K, MachowskaMajchrzak A, Skrzypek M. Fatigue and its association with sleep disorders, depressive symptoms and anxiety in patients with multiple sclerosis. Neurol Neurochir Pol. 2012;46(4):309-317.

68. Su JB, Yang XH, Zhang XL, et al. The association of long-term glycaemic variability versus sustained chronic hyperglycaemia with heart rate-corrected QT interval in patients with type 2 diabetes. PLoS One. 2017;12(8):e0183055.

69. Johnson J, Louch G, Dunning A, et al. Burnout mediates the association between depression and patient safety perceptions: a cross-sectional study in hospital nurses. J Adv Nurs. 2017;73(7):1667-1680.

70. Stirratt MJ, Dunbar-Jacob J, Crane HM, et al. Self-report measures of medication adherence behavior: recommendations on optimal use. Transl Behav Med. 2015;5(4):470-482.
Patient Preference and Adherence

\section{Publish your work in this journal}

Patient Preference and Adherence is an international, peer-reviewed, open access journal that focuses on the growing importance of patient preference and adherence throughout the therapeutic continuum. Patient satisfaction, acceptability, quality of life, compliance, persistence and their role in developing new therapeutic modalities and compounds to optimize

\section{Dovepress}

clinical outcomes for existing disease states are major areas of interest for the journal. This journal has been accepted for indexing on PubMed Central. The manuscript management system is completely online and includes a very quick and fair peer-review system, which is all easy to use. Visit http://www. dovepress.com/testimonials.php to read real quotes from published authors. 